\title{
Epílogo: interpretación jurídica y direcciones de ajuste
}

Juan Ruiz Manero

Universidad de Alicante

Riccardo Guastini es uno de los teóricos del Derecho que han ejercido una influencia mayor sobre mí. Esta afirmación no implica, desde luego, que yo sea un seguidor de Guastini (en el sentido de concordar con sus principales tesis, de que mi propio trabajo se desarrolle en el marco de la teoría del Derecho elaborada por él, etc.). Si yo dijera ser, en este sentido o en alguno análogo, seguidor de Guastini, ello no solo sería obviamente falso, sino sería, sobre todo, una afirmación que, estoy seguro, el propio Riccardo rechazaría con horror. Pero no siempre la influencia de alguien sobre otro se traduce en que el segundo sea seguidor del primero. Alguien puede haber ejercido una gran influencia sobre otro precisamente porque este último haya tendido muy frecuentemente a construir sus propias aportaciones no solo apoyándose en el primero, tomándolo como punto de partida, sino también adoptándolo como alguien contra el cual pero con ayuda de cuyos propios instrumentos se piensa. $\mathrm{Y}$ tal es el caso, creo, de la influencia que Riccardo Guastini ha ejercido sobre mí. Influencia que ha tenido 
invariablemente como punto de partida la costumbre de estudiar, antes de ocuparme de cualquier problema de teoría del Derecho, los textos que sobre ese problema hubiera escrito Riccardo. Y que ha pasado, también invariablemente, por adoptar instrumentos (enfoques, conceptos, distinciones, etc.) proporcionados por Riccardo para, muy a menudo, utilizarlos para abordar el problema de manera incompatible con los lineamientos generales del propio Riccardo, a quien venía a asumir, de manera más o menos expresa en algunos casos y tácita en otros, como interlocutor-antagonista.

\section{2}

Tal cosa es, precisamente, lo que en esta ocasión he de hacer de forma expresa y ello por la propia configuración de este número de Discusiones, destinado a discutir el trabajo -"El escepticismo ante las reglas replanteado" - que contiene la última formulación guastiniana de su teoría de la interpretación. Esta última formulación resulta, como todas las suyas, perfectamente clara y ordenada y ello, sin duda, facilita enormemente las cosas al crítico. Mis observaciones a la misma afectarán, primero, a la propia manera de cómo en ese texto se traza la tipología de las teorías de la interpretación jurídica; segundo, a algunas distinciones (o a algunos aspectos de algunas distinciones) contenidas en él; tercero, a la manera en que Riccardo presenta en este texto la diferencia entre su propia concepción y la "teoría de la vigilia". Propondré muy sucintamente, para terminar, algunos lineamientos de una visión de la interpretación alternativa a la de Riccardo.

\section{3}

Pero antes de ocuparnos de esta última versión de la teoría de la interpretación de Guastini es conveniente, me parece, despejar el terreno en relación con algo -la llamada concepción "escéptica radical" de la

${ }^{1}$ Riccardo Guastini: "El escepticismo ante las reglas replanteado", en este mismo número de Discusiones. 
Epílogo: interpretación jurídica y...

interpretación- que se presenta como un pariente cercano de la concepción guastiniana. Si esta última se presenta como una teoría "escéptica moderada" de la interpretación, calificar la propia teoría de la interpretación de "escéptica radical", como hace Michel Troper ${ }^{2}$, parece indicar que una y otra son especies (moderada una, radical la otra) del mismo género, el escepticismo interpretativo. Pues bien: en mi opinión, el llamado "escepticismo radical" de Troper no solo no comparte un género común con el "escepticismo moderado" representado aquí por Guastini, sino que ni siquiera es propiamente una teoría de la interpretación jurídica (aun reduciendo el alcance de ésta, conforme con las pretensiones del propio Troper, a la interpretación judicial). Y no lo es, a mi juicio, porque las razones que aduce Troper en apoyo de su posición tienen que ver con lo que institucionalmente le resulta posible hacer al juez (o, si se prefiere decirlo de esta otra forma, con los poderes normativos del juez), pero no con su posición vinculada en relación con las emisiones de las autoridades edictoras de normas (el legislador, en sentido amplio). Y es en este último terreno, a mi juicio, y no en el de las posibilidades institucionales o poderes normativos del juez, en el que ha de ubicarse una teoría de la interpretación judicial. Pues hablar de interpretación judicial tiene como presupuesto la idea de que el juez ha de fundamentar sus resoluciones en estándares que le preexisten y que están investidos de autoridad para el juez en función de su fuente. La interpretación judicial consiste centralmente en la identificación del contenido de esos estándares a partir de las formulaciones provenientes de las autoridades normativas. Y, como muy acertadamente dice el mismo Guastini en el texto que discutimos, "la interpretación [...] posee límites (conceptuales, no fácticos), en el sentido de que no todo enunciado que pretende ser una interpretación puede razonablemente ser subsumido bajo el concepto de interpretación. Interpretar no consiste en atribuir cualquier significado, sino en atribuir uno de los significados que se encuentran dentro del rango de significados admisibles según (a) el uso común, (b) los métodos

2 Michel Troper: "Anotaciones sobre Guastini", en este mismo número de Discusiones. 
interpretativos en uso y, (c) las teorías dogmáticas". Y del mismo modo que no todo lo que pretende ser interpretación puede razonablemente subsumirse dentro del concepto de interpretación, tampoco todo lo que pretende ser una teoría de la interpretación puede razonablemente subsumirse bajo ese concepto. Por así decirlo: son disputas ubicables en el ámbito de las teorías de la interpretación las que enfrentan, por un lado, a quienes sostienen, como viene a ser el caso de Guastini, que a los textos jurídicos pueden adscribírseles diversos significados igualmente admisibles, de forma que no cabe sino realizar una opción no susceptible de fundamentación por alguno de ellos y a quienes defienden, por el contrario, que las disputas interpretativas presuponen precisamente, para su inteligibilidad misma, la idea de que alguno de esos significados es correcto; pero se sitúa, en cambio, fuera de lo que puede razonablemente cubrirse con el término "teoría de la interpretación" una concepción, como la de Troper, que sostiene que, como él mismo dice, no hay "desde un punto de vista jurídico, ninguna diferencia entre una norma resultado de una genuina interpretación y una norma resultado de un acto de creación", de lo que se deriva que "si el intérprete, por ejemplo una corte suprema, puede dar a un enunciado el significado que quiera, no es algo que suceda de facto, sino que tiene lugar porque el intérprete ha recibido la competencia y porque el orden jurídico hace que sus decisiones posean efectos jurídicos [...] La teoría [escéptica radical] constata que el orden jurídico, al otorgar a un intérprete la última palabra, le otorga también el poder de dar a los textos el significado que quiera". A este respecto cabe decir, en mi opinión, primero, que Troper tiene parcialmente razón en estas observaciones; segundo, que ese tener razón, pero solo parcialmente, tiene que ver con la ambigüedad de "puede", incluso en contextos relativos al sistema jurídico y, tercero, que el "puede" relevante aquí no es aquel que Troper tiene en mente. Veamos todo ello: Troper tiene, desde luego, razón en que el sistema jurídico no subordina el que las sentencias desplieguen sus efectos jurídicos a que esas sentencias sean regulares, esto es, a que tengan como fundamento una norma (o, si se prefiere decirlo así, una interpretación admisible de una disposición, o de un fragmento de disposición o de un conjunto de disposiciones o de fragmentos de disposiciones) en la que 
Epílogo: interpretación jurídica y...:

sean subsumibles los hechos del caso y que el juez tenga el deber de usar como tal fundamento. Las sentencias despliegan sus efectos jurídicos aun cuando sean irregulares, esto es, aun cuando no tengan como fundamento una norma (o, de nuevo, una interpretación admisible de una disposición, etc.) así. Y, si lo único que quiere decir es eso, Troper tiene claramente razón en que el que "el intérprete, por ejemplo una corte suprema, puede dar a un enunciado el significado que quiera, no es algo que [meramente] suceda de facto": pues las sentencias de un tribunal despliegan sus efectos simplemente porque al tribunal de que se trate le haya correspondido, de acuerdo con su competencia, enjuiciar el caso, y despliegan esos efectos con carácter definitivo si ese tribunal es, para el caso, de última instancia. El "puede" aquí relevante no alude, pues, a una posibilidad meramente fáctica, sino a una posibilidad institucional: "puede" significa aquí, y este es el punto en el que Troper tiene razón, "le es posible (o, si se prefiere, 'tiene la capacidad de' o 'el poder normativo para') producir los correspondientes efectos jurídicos”. Pero el lugar propio de la teoría de la interpretación judicial no es el de las posibilidades institucionales del juez (es decir, el de lo que el juez puede hacer en el sentido de tener capacidad o poder normativo para producir los correspondientes efectos jurídicos) sino el de sus vínculos deónticos: el de lo que el juez debe o puede (en sentido deóntico, en sentido permisivo) hacer. Pues una teoría de la interpretación judicial, para merecer tal nombre, tiene que tomar como punto de partida el deber del juez de usar ciertas normas como fundamento de sus resoluciones y ha de referirse a los deberes y a los permisos que gravitan sobre la actividad del juez consistente en establecer el sentido de las disposiciones en que se expresan aquellas normas. Y me parece que Troper, o bien prescinde de este sentido permisivo de "puede", asimilándolo indebidamente al sentido de la "posibilidad institucional", o bien considera que el tener la posibilidad institucional de hacer algo implica el permiso para hacer ese algo: lo que es, evidentemente, un non sequitur que equivale, si se trata de tribunales de última instancia, a la falacia que Hart pusiera de manifiesto, consistente en considerar que la definitividad de las decisiones de esos tribunales implica la infalibilidad (o la corrección deóntica) de las mismas. 


\section{4}

Pero ocupémonos ya de la teoría de Guastini. Empecemos por su tipología de las teorías interpretativas y, examinemos a continuación la manera cómo traza dos distinciones: la que media entre interpretación "cognitiva", por un lado, e interpretaciones "decisoria" y "creativa", por otro y la que diferencia entre interpretación "en abstracto" (u orientada a los textos) e interpretación "en concreto" (u orientada a los hechos).

4.1. En cuanto a su tipología de las teorías interpretativas, Guastini distingue, al modo ya habitual, entre teorías del "noble sueño", de la "pesadilla" y de la "vigilia" 3 . De acuerdo con la primera, los textos jurídicos se encontrarían provistos de un significado único y determinado, a propósito del cual cabe formular enunciados verdaderos o falsos. La interpretación se concibe como una actividad cognitiva que ha de desembocar en la formulación de enunciados interpretativos verdaderos, en cuanto correspondientes al significado prexistente del texto de que se trate. La teoría de la pesadilla asume, por su parte, que los textos no tienen significado alguno antes de la interpretación, sino que tal significado es el resultado de la actividad interpretativa, que no sería de ningún modo una empresa cognitiva, sino volitiva y decisional. De acuerdo, en fin, con la teoría de la vigilia, que puede, indica Guastini, ser reconducida a Hart, habría que distinguir entre casos fáciles (aquellos en los que los hechos caen con seguridad bajo el ámbito de aplicación de los 'términos clasificatorios generales' o predicados utilizados por la disposición jurídica de que se trate) y los casos difíciles (aquellos en los que, por la vaguedad o textura abierta de estos mismos predicados, es

${ }^{3}$ Las expresiones "noble sueño" y "pesadilla" provienen de Hart. Cfr. H.L.A. Hart: "American Jurisprudence through English Eyes: The Nightmare and the Noble Dream" (1977), ahora en H.L.A. Hart: Essays in Jurisprudence and Philosophy, Clarendon Press, Oxford, 1983. La expresión "vigilia", por lo que sé, fue utilizada por primera vez en este sentido por José Juan Moreso: La indeterminación del Derecho y la interpretación de la Constitución, Centro de Estudios Políticos y Constitucionales, Madrid, 1997. 
Epílogo: interpretación jurídica y...:

dudosa o discutible su aplicabilidad a los hechos del caso, que caerían bajo la zona de penumbra de su significado). Pues bien, creo que a propósito de esta tipología caben dos observaciones. La primera, y menos importante, es que quizás no hace entera justicia a la "vigilia" hartiana, pues presenta únicamente la versión primera (cronológicamente) de la misma. Quiero decir lo siguiente: en lo que podríamos llamar el primer Hart, el Hart de "Positivism and the Separation of Law and Morals" 4 y de The Concept of Law", la cuestión de si un caso es fácil o difícil es exactamente la misma cuestión de si el caso se encuentra cubierto por el núcleo de significado establecido, de acuerdo con las convenciones lingüísticas vigentes, de los predicados utilizados en la formulación de una regla o si, por el contrario, resulta dudoso, teniendo en cuenta esas mismas convenciones, que se encuentre o no cubierto por ese mismo significado establecido. Pero a partir del artículo de 1967 "Problems of the Philosophy of Law" la identificación entre ambas cuestiones desaparece, pues, dice ahora Hart, "los significados de las palabras pueden ser claramente controlados por referencia al propósito de una disposición legislativa, el cual propósito puede tanto haber sido explícitamente establecido como considerarse como tal en virtud de la existencia de un acuerdo general al respecto" . $^{\circ}$ Esto, obviamente, implica que ya no pueden ser consideradas como equivalentes las dos cuestiones a las que se acaba de hacer referencia: pues un caso que, de acuerdo con las convenciones lingüísticas compartidas, aparezca como un caso difícil puede resultar (en virtud del propósito explícitamente indicado o generalmente atribuido a la regla) un caso fácil; y, a la inversa, un caso que, de acuerdo con las convenciones lingüísticas, aparezca como fácil, al estar claramente incluido en el núcleo de significado de los términos empleados en la formulación de la regla, puede resultar difícil (pues resulte dudoso si la

${ }^{4}$ H.L.A. Hart: "Positivism and the Separation of Law and Morals" (1958), ahora en H.L.A. Hart: Essays in Jurisprudence and Philosophy, cit.

${ }^{5}$ H.L.A. Hart: The Concept of Law ( $1^{\text {a }}$ ed.), Oxford University Press, 1961.

${ }^{6}$ H.L.A. Hart: "Problems of the Philosophy of Law" (1967), ahora en H.L.A. Hart: Essays in Jurisprudence and Philosophy, cit., p. 106. 
aplicabilidad de la regla a él resulta concordante con el propósito expreso o atribuido de forma general a esa misma regla).

Pero esta es, desde luego, una cuestión menor. Mayor importancia tiene, me parece, la observación que sigue: la tipología de las teorías de la interpretación, tal como la presenta Riccardo, parece dar por descontado que la única dirección de ajuste relevante para los enunciados interpretativos es la que va del presente al pasado, o si se prefiere decirlo así, de palabras (los enunciados interpretativos) a mundo (aun cuando se trate aquí de un fragmento del mundo compuesto también por palabras: los textos jurídicos) y no la que va del futuro (las decisiones aplicativas) al presente (los enunciados interpretativos) o, si se prefiere decirlo así, de mundo a palabras. Dicho de otra manera: lo que divide a las diversas teorías de la interpretación viene a ser, en el esquema de Riccardo, si los enunciados interpretativos pueden ser verdaderos o falsos, en el sentido de correspondencia con el fragmento del mundo constituido por los textos jurídicos. La teoría del noble sueño afirmaría que los enunciados interpretativos tienen siempre valor de verdad, la de la pesadilla que no lo tienen nunca, y la de la vigilia que lo tienen en algunos casos (los fáciles) y no en otros (los difíciles). Pero lo único relevante sería, para las tres concepciones, si hay posibilidad de verdad en este sentido de correspondencia. Y si esto es lo único relevante quedan obviamente fuera de la tipología aquellas concepciones de la interpretación que no ven la misma en términos de descubrimiento (posible siempre, de acuerdo con el noble sueño, siempre imposible, de acuerdo con la pesadilla, posible en algunos casos e imposible en otros, de acuerdo con la vigilia) de significados prexistentes, sino que entienden que la empresa interpretativa se orienta a justificar (y no a descubrir) la adscripción de significados que se consideran correctos como fundamento de las decisiones aplicativas futuras. Para una concepción de la interpretación de este género, la dirección de ajuste más relevante de los enunciados interpretativos es la que va del futuro (las decisiones aplicativas) al presente (los propios enunciados interpretativos) o, si se quiere, la que va de mundo a palabras. Y el ajuste de presente a pasado, de palabras (los enunciados interpretativos) a mundo (los textos jurídicos) es, en estas concepciones, solo un 
Epílogo: interpretación jurídica y...

ingrediente de un proceso argumentativo complejo que se entiende como orientado a proporcionar fundamentos justificados para las decisiones aplicativas futuras: piénsese, en este sentido, en el papel de la dimension of fit -esto es, de la correspondencia de los enunciados interpretativos con los materiales jurídicos- en una concepción de la interpretación como la dworkiniana ${ }^{7}$ (y la referencia a Dworkin tiene únicamente el sentido de poner un ejemplo, entre muchos posibles, del tipo de teoría de la interpretación a que me estoy refiriendo). Quiero decir, en definitiva, que la tipología de Riccardo deja fuera a toda la familia de concepciones de la interpretación que entienden ésta no como conocimiento de realidades (significados) prexistentes sino como propuesta de fundamentos justificados para las decisiones futuras, pero que entienden, a la vez, que esta tarea de proponer fundamentos justificados es una empresa racionalmente controlable: o, dicho de otro modo, que hay propuestas objetivamente correctas (o verdaderamente correctas, si así lo preferimos) y otras que no lo son. Y dejar fuera a toda la familia de concepciones de la interpretación de este género es, a mi juicio, mucho dejar fuera. Tanto como para poner en cuestión el criterio clasificatorio que Riccardo hace suyo.

4.2. El núcleo de la teoría neokelseniana de la interpretación que Guastini viene a proponernos reside, a mi modo de ver, en la distinción entre "interpretación cognitiva", de un lado, e "interpretación decisoria" e "interpretación creativa", por otro. La interpretación cognitiva, nos dice Guastini al modo de Kelsen, "consiste en identificar, sin elegir entre ellos, los posibles y diferentes significados de un texto normativo". En este punto, la diferencia que aporta Guastini frente al planteamiento kelseniano reside en que el marco de admisibilidad no viene definido únicamente, como parece ocurrir en Kelsen, por consideraciones lingüísticas: los significados admisibles, señala Guastini, vienen determinados, desde luego, por "las reglas lingüísticas -sintácticas, semánticas y pragmáticas- compartidas", pero también por "las distintas técnicas interpretativas en uso y las tesis dogmáticas difundidas en

${ }^{7}$ R. Dworkin: Law's Empire, Harvard University Press, 1986. 
doctrina". Pero, a partir de aquí, todo es puro Kelsen, empezando por la consideración de que "la interpretación cognitiva, tal como sugiere el mismo nombre, es una operación puramente cognoscitiva privada de cualquier efecto práctico". Y si la interpretación cognitiva es puramente cognoscitiva, las interpretaciones decisoria y creativa son operaciones "políticas" y como tales, también al modo de Kelsen, puramente decisionales: la llamada interpretación "creativa" consiste en adscribir un significado situado fuera del perímetro de los admisibles, en tanto que la interpretación decisoria consiste en "elegir" uno de los significados admisibles, descartando los restantes. En la imposibilidad de fundamentar racionalmente la opción por alguno de estos significados admisibles es donde reside, señala Guastini, "el verdadero fundamento del escepticismo ante las reglas": en "el reconocimiento de que es siempre posible interpretar un mismo texto de múltiples formas distintas, asociado con la tesis de que no existe un criterio de verdad para los enunciados interpretativos". De esta forma, el "escepticismo moderado" de Guastini viene a identificarse centralmente con la teoría kelseniana de la interpretación. Ocurre, sin embargo, que, a diferencia de lo que sucede con Guastini, la teoría kelseniana de la interpretación se presenta a sí misma como una teoría prescriptiva de la interpretación. Kelsen no pretende en ningún momento que su teoría de la interpretación refleje las prácticas interpretativas de los juristas teóricos y prácticos; pretende, bien al contrario, reorientar esas prácticas de forma que resulte una práctica interpretativa que se adecue, para los juristas teóricos, a las que él considera como exigencias de cientificidad y que, para los órganos de aplicación, sea consciente del carácter decisional -y, por ello, desde su concepción, no fundamentable racionalmente- de sus opciones interpretativas. Pero el caso de Guastini es, a este respecto, bien distinto, pues su teoría de la interpretación jurídica no pretende ser una teoría prescriptiva, sino, por el contrario, una "reconstrucción racional de la práctica de atribución de significado llevada a cabo por los intérpretes jurídicos". Y, partiendo de esta pretensión, no puede dejar de señalarse que la teoría guastiniana de la interpretación es paradójica, o, si se prefiere decirlo así, autorrefutatoria. Y ello porque es claro que "la práctica de atribución de significado llevada a cabo por los intérpretes jurídicos" no corresponde en modo 
Epílogo: interpretación jurídica y...

alguno al modelo de Guastini: no hay ningún jurista teórico de alguna relevancia que considere, no ya que su trabajo, en la medida en que es conocimiento, deba limitarse a presentar un elenco de los diferentes significados posibles, sino ni siquiera que piense que la elaboración de tal elenco constituya una de las tareas que debe llevar a cabo; y no hay tampoco ningún órgano de aplicación que presente sus opciones interpretativas como fruto de una decisión discrecional entre diversos significados igualmente admisibles (parece claro que si hiciera tal cosa incurriría en alguna suerte de inconsistencia pragmática). Dicho de otro modo: la teoría de la interpretación de Guastini implica que la visión que los juristas tienen de su propia práctica interpretativa es engañosa, de forma deliberada $\mathrm{o}$, más probablemente, inconsciente. No veo ningún sentido en el que pueda considerarse como "reconstrucción racional" de una práctica un discurso que presenta esa práctica como centralmente errada. Si, como es el caso, los juristas (y ello vale tanto para los dogmáticos como para los aplicadores) creen que su tarea, en punto a interpretación, consiste precisamente en justificar qué significado debe atribuirse a las formulaciones normativas, no consigo ver cómo puede sostenerse, como hace Riccardo, que la cuestión de "qué significado debe ser elegido -dentro del rango de los significados posibles- es una cuestión normativa, irrelevante desde el punto de vista de una teoría descriptiva de la interpretación"; teniendo en cuenta, sobre todo, que una teoría descriptiva de la interpretación no es otra cosa, según el propio Riccardo (y acudo aquí a otra formulación de una idea que en el texto de Riccardo se encuentra una y otra vez), que una "reconstrucción de la práctica efectiva de una determinada comunidad interpretativa". En algún momento de su réplica ${ }^{8}$ dice Riccardo que quien no vea la diferencia entre interpretación cognitiva e interpretación decisoria "es ciego". Yo debo padecer serios problemas de visión, pues si bien veo la diferencia entre la interpretación doctrinal y la llevada a cabo por un órgano de aplicación, no creo que la primera sea puramente cognitiva de marcos de significado preexistentes y que la segunda añada a ello un momento decisional insusceptible de fundamentación racional. Creo

${ }^{8}$ Riccardo Guastini: "Réplica", en este mismo número de Discusiones. 
que entre ambas hay una línea de continuidad que consiste en que tanto una como otra tratan de justificar como correcta la atribución de significados que proponen. Sobre ello volveré brevemente más tarde.

4.3. $\mathrm{Y}$ vayamos ahora a la distinción guastiniana entre interpretación dirigida a textos (o "en abstracto" o "de enunciados completos") e interpretación dirigida a hechos (o "en concreto" o "de predicados"). También aquí nos dice Riccardo en su réplica que "si alguno no ve la diferencia entre los problemas" correspondientes a una y otra "es ciego". Y también aquí he de concluir que, si no ceguera, sí debo padecer algún problema de visión, porque no acabo de ver la manera cómo Riccardo presenta la interpretación "en concreto" o "de predicados". Creo que el concepto guastiniano de problemas de interpretación "en concreto" trata de cubrir los mismos problemas que el concepto de MacCormick de "problemas de calificación"9 (que él opone a los "problemas de interpretación") o que el concepto de Alchourrón y Bulygin de "lagunas de reconocimiento" 10 , esto es, problemas relativos a la subsumibilidad de hechos, o de entidades, en los predicados de normas. A este respecto, Riccardo, en su artículo inicial parece circunscribir los problemas de subsumibilidad a la subsunción "individual" (la inclusión de un hecho o entidad individual en una clase) "sin ni siquiera mencionar -como reconoce él mismo en la réplica- la llamada subsunción "genérica" (la inclusión de una clase dentro de otra clase más amplia) cuya ausencia le había sido señalada, muy justamente en mi opinión, por Isabel Lifante en su comentario ${ }^{11}$. Pues bien, lo que, por mi parte, quiero poner de manifiesto es lo siguiente: no hay problemas de subsumibilidad individual que puedan tratarse de manera autónoma respecto de los problemas de subsumibilidad genérica. Por lo siguiente: porque no puede fundamentarse la opción por incluir, o por no incluir, una

${ }^{9}$ Neil MacCormick: Legal Reasoning and Legal Theory, Clarendon Press, Oxford, 1978.

${ }^{10}$ Carlos Alchourrón y Eugenio Bulygin: Introducción a la metodología de las ciencias jurídicas y sociales, Astrea, Buenos Aires, 1974.

${ }^{11}$ Isabel Lifante: "Distinciones y paralogismos. A propósito del escepticismo guastiniano", en este mismo número de Discusiones. 
Epílogo: interpretación jurídica y...:

determinada entidad o hecho individual en una clase más que aludiendo a las propiedades de esa entidad o hecho y a si esas propiedades corresponden o no a las de la clase de referencia. Aclararé lo que quiero decir con dos ejemplos ya muy clásicos: no hay manera de contestar fundadamente a la pregunta de si el contrato que Tim y Tom celebraron el domingo pasado es o no es, por esa circunstancia, sacrílego, más que determinando si la propiedad (descriptiva) "celebrado en domingo" es subsumible o no en la propiedad (valorativa) "sacrílego"; y si nos preguntamos si la bicicleta que Riccardo compró la semana pasada o la bicicleta con la que Juan ha paseado hoy deben considerarse como vehículos, no podemos contestar fundadamente a ello más que determinando si las propiedades que determinan que algo sea una bicicleta son subsumibles en las propiedades que determinan que algo sea un vehículo. Parece, pues, que la subsunción individual se fundamenta siempre en alguna subsunción genérica. Y esta última, la subsunción genérica es, como reconoce el propio Riccardo en su réplica, "parte esencial de la interpretación en abstracto". Esto, naturalmente, no niega por completo la distinción entre problemas de interpretación "en abstracto" y problemas de interpretación "en concreto", pero tiene el efecto de relativizarla (o, como reconoce el propio Riccardo en su réplica, de "difuminarla" [sfumarla]) bastante.

\section{5}

La relativiza o la difumina hasta el punto de que es dudoso que, si lo anterior es correcto, haya realmente una diferencia de alguna relevancia entre la teoría de la "vigilia" y la de Riccardo. En el texto inicial de éste encontramos lo que sigue:

"Según la teoría de la vigilia, parecería que la controversia entre cognitivismo y escepticismo ante las reglas recae sobre la interpretación dirigida a hechos, esto es, sobre el estatuto lógico de los enunciados subsuntivos [...]. Yo creo que esto no es así. El objeto de la controversia son en realidad los enunciados interpretativos en sentido estricto, enunciados con la forma ' $T$ significa $S$ ' y que pertenecen a la interpretación dirigida a textos. Que los enunciado subsuntivos pueden 
tener valor de verdad es una tesis admisible (si bien bajo ciertas condiciones). Pero una buena teoría de la interpretación ha de proporcionar una respuesta convincente a la cuestión sobre la posibilidad de que los enunciados interpretativos tengan valor de verdad".

Ahora bien: si se reconoce, como hace aquí Riccardo, que los enunciado subsuntivos, como sostiene la teoría de la vigilia, pueden tener valor de verdad bajo ciertas condiciones, si se admite que, como he tratado de mostrar, todo enunciado subsuntivo individual depende de un enunciado subsuntivo genérico, y si se reconoce, como hemos visto que hace el propio Riccardo en su réplica, que la subsunción genérica es parte esencial de la interpretación en abstracto o dirigida a textos ¿qué más se requiere para determinar que los enunciados interpretativos, como los subsuntivos, tienen valor de verdad bajo ciertas condiciones? Y siendo las cosas así, ¿qué diferencia resta entre la teoría hartiana de la vigilia y la de Riccardo Guastini?

\section{6}

Antes (en 4.1) he objetado a la tipología de las teorías interpretativas presentada por Riccardo que ésta depende de un criterio que presupone que la dirección de ajuste de los enunciados interpretativos era de palabras (los propios enunciados interpretativos) a mundo (el lenguaje del legislador) y he mostrado que esto deja fuera a todas las concepciones de la interpretación que entienden que la principal dirección de ajuste de los enunciados interpretativos es de mundo (las decisiones aplicativas futuras) a palabras (los enunciados interpretativos). En mi opinión, una teoría de la interpretación que pretenda reconstruir las prácticas interpretativas reales de la comunidad jurídica ha de partir de esto y de considerar que el ajuste de palabras (los enunciados interpretativos) a mundo (el lenguaje del legislador) es solo una condición necesaria de la corrección de los enunciados interpretativos, que deben entenderse como enunciados prácticos, como enunciados dirigidos no a conocer realidades prexistentes, sino a dirigir acciones futuras. 
Epílogo: interpretación jurídica y...

A mi modo de ver, parece razonable ubicar bajo el rótulo "interpretación", todo el proceso argumentativo que permite pasar de las normas emitidas por el legislador a la norma que opera como premisa mayor de un razonamiento aplicativo. Con la referencia a "todo el proceso argumentativo" quiero indicar que me parece razonable ubicar bajo el mismo rótulo tanto los razonamientos dirigidos a la determinación del significado de las formulaciones emitidas por el legislador (lo que, en el lenguaje de Guastini, sería propiamente interpretación) como los razonamientos que tienen como objeto la construcción de balances entre principios, la determinación de la ratio legis, la solución de antinomias, la integración de lagunas, etc. (todo aquello que, en el lenguaje de Guastini, sería construcción jurídica). Por dos razones: primero, por las interconexiones evidentes entre ambos tipos de razonamientos: qué significado adscribamos a las formulaciones del legislador puede depender, por ejemplo, de qué entendamos que constituye la ratio legis; que un cierto caso constituya o no una laguna o que dos normas sean o no antinómicas entre sí puede depender, también por ejemplo, del significado que hayamos adscrito a ciertas emisiones del legislador; y, segundo (y ésta es, a mi juicio, la razón más importante para una consideración unitaria) porque ambos tipos de razonamientos se orientan a la misma finalidad: pasar de las normas emitidas por el legislador a la norma capaz de operar como premisa mayor de un razonamiento aplicativo. Se habrá observado que hablo de "pasar de las normas emitidas por el legislador a la norma capaz de operar como premisa mayor de un razonamiento aplicativo" y no de "pasar de las disposiciones (o de las formulaciones) a las normas". Pues, a mi juicio, el legislador emite normas, y no meros enunciados o disposiciones: si no fuera así, no tendría sentido calificarlo como autoridad normativa. Ocurre, sin embargo, que las normas emitidas por el legislador necesitan de un trabajo de transformación para obtener, a partir de ellas, normas que puedan servir como fundamento para la resolución de un caso. Por las razones conocidas: las normas emitidas por el legislador pueden contener lagunas o antinomias, pueden no precisar (si se trata de principios) sus condiciones de aplicación, pueden presentar problemas de ambigüedad y vaguedad que deban ser reducidos de acuerdo con las 
exigencias del caso. Pero también por otra razón en la que se suele reparar menos: y es que, incluso cuando la norma que se utiliza como fundamento para la resolución del caso se expresa mediante la misma formulación utilizada por el legislador, el tránsito de la segunda a la primera implica, como puso de relieve Josep Aguiló ${ }^{12}$, una transformación capital: pues la norma emitida por el legislador puede estar afectada por excepciones implícitas que no resultan enteramente anticipables y ello supone que los deberes estipulados por ella son meramente prima facie; mientras que para fundamentar la resolución del caso necesitamos, en cambio, una norma que establezca deberes concluyentes.

Ver así las cosas supone plantear de otra manera la relación entre interpretación doctrinal e interpretación de los órganos de aplicación. La primera no ha de entenderse como interpretación puramente cognoscitiva desprovista de efectos prácticos, ni como enumeración de todos los significados posibles. La interpretación doctrinal se encamina de hecho, y así debe hacerlo, a justificar la adscripción de un determinado significado a las emisiones del legislador, así como a realizar aquellas operaciones (de balance entre principios, de integración de lagunas, de solución de antinomias) igualmente necesarias para desembocar en la norma-premisa del razonamiento aplicativo. En este sentido, la doctrina cumple una función de intermediación entre las emisiones normativas en bruto de las autoridades normativas y la aplicación judicial del Derecho. Utilizando algo libremente terminología de Carlos Alchourrón ${ }^{13}$, diríamos que la elaboración doctrinal del Derecho trata de avanzar tanto como sea posible del Libro Maestro (de las fuentes) a un Sistema Maestro (compuesto por normas aptas para operar como premisa mayor de un razonamiento aplicativo)

12 Josep Aguiló: Teoría general de las fuentes del Derecho (y del orden jurídico), Ariel, Barcelona, 2000, pp. 106-107.

13 Carlos Alchourrón: "Sobre Derecho y lógica" (1996), ahora en Carlos Alchourrón: Fundamentos para una teoría general de los deberes, edición y estudio introductorio de José Juan Moreso y Jorge Luis Rodríguez, Marcial Pons,

218 Madrid-Barcelona-Buenos Aires, 2010. 
Epílogo: interpretación jurídica y...

determinado, coherente y completo. Tanto como sea posible: porque la incapacidad humana de anticipar por completo las combinaciones de propiedades que puedan presentar los casos futuros hace que nunca pueda excluirse que, aun tras la mejor elaboración doctrinal, el sistema siga conteniendo antinomias (del tipo que Alf Ross denominaba "parcialparcial"14, o Carlos Alchourrón "de inconsistencia condicional"15), lagunas o indeterminaciones. Antinomias, lagunas o indeterminaciones que descubrimos cuando el caso individual presenta combinaciones de propiedades que ni el legislador ni los juristas teóricos habían previsto.

${ }^{14}$ Alf Ross: Sobre el Derecho y la justicia (1958), trad. de Genaro R. Carrió, Editorial Universitaria de Buenos Aires, 1970 -2 ${ }^{\mathrm{a}}$ ed.-, pp. 125.

${ }^{15}$ Carlos Alchourrón: "Conflictos de normas y revisión de sistemas normativos" (1988), ahora en Carlos Alchourrón y Eugenio Bulygin: Análisis lógico y Derecho, Centro de Estudios Constitucionales, Madrid, 1991. 